\title{
COMPETITIVE SPORT IN REHABILITATION
}

COCHRANE, G. M.

Dept. of Physical Medicine, Derbyshire Royal Infirmary

Any disability makes a patient conscious of the individual movements involved in performing a task and distracts him from the objective. Selected work and games divert his attention from his disability and focus it upon an activity which provides less obtrusively though no less certainly his treatment. Constant stimulation of interest, variation of methods to alleviate boredom and monotony, explanations which allow a patient intelligent appreciation of why such methods are being used, charts of progress by which the patient can follow a step by step goal to be achieved are all necessary at one time or another to promote recovery. Most of us enjoy one or more sports as serious pastimes in which our aspiration reaches beyond proficiency to excellence in the performance of that particular sport. We endeavour at each attempt to improve on our previous attainments whether it be to cover the distance in shorter time, strike the missile harder or aim it straighter. Besides striving with ourselves we vie with others, as the most fundamental necessity to produce maximum effort is some form of incentive. This is of singular importance in rehabilitation. We must instil ambition into our patients and be ourselves ambitious for them. Incentive may take the form of absorbed interest in the immediate objective, the urge to get back to gainful employment or enthusiasm of competition. We can think of patients who have had to be fit by a certain date, perhaps to qualify for a place in a team, when the time available was shorter than it commonly takes for reasonable function but they worked so well at their treatment that they succeeded. Competition distracts from the pre-occupation of inefficiency, pain and monotony. We may compete with ourselves to better our previous best and this is our common practice in circuit training or we may compete against others to draw level and, if possible, exceed them.

It will be appreciated that the application of suitable competitive sport will bear relation to the type of hospital or rehabilitation unit, its geographic situation, the average age of the patients, the facilities of equipment and space and the number of staff. In this context sports are being used as a means to restore fitness and the sport itself is of less importance. There is no room here for the purist. The hockey sticks may be inverted walking sticks, the cricket ball a tennis ball and cyclists mercilessly handicapped by vintage machines. The sport and its rules will be modified, simplified and distorted to suit the patients. New sports will be invented; in our geriatric hospital we have 
tried competitive undressing and dressing of the top clothes of hemiplegic patients and they play a dangerous team game of hockey, each team sitting side by side in a row and the two teams facing each other - far enough apart to escape injury by flailing walking sticks whilst each side endeavours to propel the beach ball towards its goal at the opposite open ends of the file. To qualify for use any sport must provide the precise treatment needed, must be controlled by the therapist and must be enjoyed. If the game is not enjoyed there is no profit from it.

Several points must be borne in mind:-

Do the participants understand the rules and objectives and if not does the teaching and instruction in the techniques justify the time spent - as in archery and fencing?

Does the sport come within or near to the capabilities of the patient?

Does he feel reasonably safe in the performance of the sport?

In the team event can the patients be evenly matched and all, preferably, of about the same capacity and proficiency?

Can the game be controlled adequately to achieve its therapeutic aims, to exhort the shirkers, encourage the diffident, restrain the bumptious, ensure that the desired movements are performed and trick movements excluded?

In a motional game, the pattern is unconsciously planned in the mind before being translated to the moving parts for performance. It is necessary to differentiate between games which are primarily concerned with building up power, tolerance and speed of movement and those, like chess and billiards, which need mental deliberation and specific pattern of movements of hand and mind. Both types of competitive games have a place in our programme. The type of sport is chosen for the patient, having regard to the nature of his disability and its specific treatment, improvement in his general health beyond the local disorder and the overall aim of rehabilitation to return to near normal life, for example confident on his artificial limb or robust in his wheelchair. For those who are permanently confined to wheelchairs, competitive sport introduced in treatment may become an absorbing interest later, and we recall the Stoke Mandeville Games for the Paralysed, archery, dartchery, basketball, fencing, field events (javelin distance and precision, shot-put, discus and club throwing), snooker, swimming, table tennis, weight lifting, wheelchair races and wheelchair slalom.

Some of the competitive sports used at a Rehabilitation Unit concerned largely in the after care of injured hands, concentrate on the importance of a normally functioning hand in earning a living, 
practising a hobby or allowing independence in daily activities needs no emphasis. In disabilities of the hand more than in any other region of the body the finest surgery and after care are essential. An engineering workshop has been erected for the early treatment of hands following injury, disease or surgical interference so that we may re-educate function on machines and with materials that are commonplace in our engineering community. Games adapted for particular movements and positioning of the hand and arm and can be used if necessary with the arm supported in a sling with spring assistance or resistance:-

Draughts encourages finger and thumb movements, power of grip, instrinsic hand re-education, wrist pronation, elbow extension and can be adapted by having boards of three sizes - normal, $2 \mathrm{ft}$. square and $3 \mathrm{ft}$. square. The size, shape and weight of the pucks range from normal to as large and heavy as possible. The magnetic variation of the game, with a sheet of steel painted as a draught board with button magnets used for draughts, makes it possible to play the game in any position from the horizontal to the vertical, including overhead and this can be useful in re-education of a radial palsy. All sorts of variations are possible, the game can be played on the floor to encourage crouching and kneeling and in treating lower limb disorders the players can sit and lift the heavy pucks with their feet.

Competitive blow football with the patients squeezing the rubber bulb of a syringe thus blowing out air which propels a ping-pong ball uses the outer and middle range of long flexors to the fingers and thumb and the extensors of the wrist and metacarpo phalangeal joints with a resistance of approximately 4 lbs. The pitch consists of a table $5^{\prime} x$ $3^{\prime}$ marked as on a football field and the bulbs are those used for drawing up distilled water or for lighter work, the bulbs used by florists for spraying flowers: 2 or 4 people can play. Fatigue is quickly built up - half a minute each way is sufficient the first time, the first $\frac{1}{4}$ minute is played with the normal hand and the second $\frac{1}{4}$ minute with the affected hand.

Flip football on the same pitch is used for extension of the metacarpo phalangeal joints and wrist. A square leather bag fits over the patient's fingers and distal part of the hand. Finger extension is used for play and the flat puck is flipped by extension of the fingers.

Flower pot and marbles encourages grip, pronation, supination, coordination and speed. A 9" flower pot is placed upside down on a wooden tray and marbles scattered around the flower pot. With a wooden mixing spoon the patient scoops up as many marbles as possible and tries to pour them through the hole in the pot within a given time.

Many other games are useful for grip and re-education of instrinsic muscles - "Pick-a-Stick", padder tennis, badminton, table tennis, quoits, darts, skittles, ring toss. 
Many of these competitive games can be used in treatment of the elbow and shoulders and we can add to them croquet and bowling. Archery has long been an important part of the treatment of paraplegics and quadriplegics at Stoke Mandeville Hospital and many other hospitals were assisted by grants by the Central Council for the Disabled about 4 years ago for the introduction of archery as a form of remedial treatment. A 25 yard range is adequate for beginners with a similar distance of clear ground behind the targets. Crush injuries of the fingers, tendon repairs, amputations of fingers (using a prosthesis when necessary) burns with contractures and tendon adhesions, Colles fractures, Sudeks atrophy and ulnar nerve palsies are examples of hand disabilities where archery has contributed to their treatment. Fractures and dislocations of the upper limbs and deltoid weakness following circumflex nerve palsy are commonly treated, starting with the wooden practice bows and progressing through the light-weight fibreglass bows, to a relatively strong one.

In treating injuries of the lower limbs, archery provides standing tolerance and walking practice and the stride stance encourages abduction at the hips for arthroplasties. The muscles of the trunk are strengthened and archery is included in the treatment programmes of those with back injuries. In the final stages of recovering hemiplegia a few progress to target shooting and of course archery is traditionally beneficial to paraplegics. Quadriplegics with active triceps can become archers. Those with some grip may need no special adaptations but without grip need an extended flexion glove to hold the bow and a hook attached to $a$ horse-hide mitten to draw back the string. Release occurs by pronation of the hand thus twisting the hook off the string. Several quadriplegics use this method and are able to compete with more able-bodied members of the community. Indoor archery is a good winter activity though a range may be only twenty yards long.

Competitive swimming is a later stage of hydrotherapy, carried out in the municipal swimming pool. After supervised remedial exercises the second half hour is a time for competition and a rough and tumble. Physically handicapped children go to the same pool on one evening a week in the summer months and their supervision is provided by volunteers swimming instructors, life-savers, policemen and cadets and those that can swim are appropriately handicapped for competition. I wish that this facility were more readily available and that swimming could be part of the treatment programme every day. In this Rehabilitation Unit there are good gymnasia and we use them and the lawns outside for football, basketball, handball, hockey, mat ball, rounders, quoits, volley ball, badminton and various other ball games. There are climbing ropes, vaulting horse and springboard and hurdles for the final stages of treatment of young men who have had menisectomies. Fencing is the most recent introduction for our Rehabilitation Officer is accomplished and sees its 
advantages. Personally I think it will only have a limited place for those who have previous experience or are particularly quick to learn new techniques though $I$ am sensible of its inherent possibilities.

Unless they be withdrawn or pre-occupied with compensation almost all patients enjoy competitive sport that is introduced correctly into their treatment programme, interestingly presented, well taught and organised. The stimulation of our enthusiasm will influence those we treat and those with whom we work. 